\title{
ON A CLASS OF NONHOMOGENEOUS ELLIPTIC PROBLEMS INVOLVING EXPONENTIAL CRITICAL GROWTH
}

\author{
Manassés de Souza - Everaldo de Medeiros - Uberlandio Severo
}

\begin{abstract}
In this paper we establish the existence of solutions for elliptic equations of the form $-\operatorname{div}\left(|\nabla u|^{n-2} \nabla u\right)+V(x)|u|^{n-2} u=g(x, u)+\lambda h$ in $\mathbb{R}^{n}$ with $n \geq 2$. Here the potential $V(x)$ can change sign and the nonlinearity $g(x, u)$ is possibly discontinuous and may exhibit exponential growth. The proof relies on the application of a fixed point result and a version of the Trudinger-Moser inequality.
\end{abstract}

\section{Introduction and the main result}

In this paper we consider the existence of nontrivial weak solutions for the following class of elliptic problems:

$$
-\operatorname{div}\left(|\nabla u|^{n-2} \nabla u\right)+V(x)|u|^{n-2} u=g(x, u)+\lambda h \quad \text { in } \mathbb{R}^{n},
$$

where $n \geq 2, \lambda$ is a positive parameter, $g$ and $V$ are functions satisfying mild conditions and $h$ belongs to the dual of an appropriated function space.

When $n=2$, problem (1.1) is related to the existence of standing wave solutions for the nonlinear Schrödinger equation

$$
i \hbar \frac{\partial \psi}{\partial t}=-\frac{\hbar}{2 m} \Delta_{x} \psi+V(x) \psi-g(x,|\psi|) \psi-h(x) e^{i \omega t},
$$

2010 Mathematics Subject Classification. 35J92, 47H10, 35B33.

Key words and phrases. Critical growth, Trudinger-Moser inequality, fixed point result, discontinuous nonlinearity.

Research partially supported by the National Institute of Science and Technology of Mathematics INCT-Mat, CNPq grants 307400/2009-3 and 308339/2010-0. 
where $m$ and $\hbar$ are positive constants, $\omega \in \mathbb{R}$ and $\psi: \mathbb{R}^{2} \times \mathbb{R} \rightarrow \mathbb{C}$. Such equations arise in various branches of mathematical physics (see for example [3], [4], [27]) and they have been the subject of extensive study during the last years.

We point out that the existence of solutions for problem (1.1) has been discussed under various conditions on the potential $V$. We refer the reader to [13], [15], [18], [30] and references therein. It is worthwhile to remark that in these works different hypotheses are assumed on $V$ in order to overcome the problem of "lack of compactness", typical for elliptic problems in unbounded domains. More precisely, in many papers it is usually assumed that the potential is uniformly positive, that is, $V(x) \geq V_{0}>0$ for any $x \in \mathbb{R}^{n}$ and satisfies one of the following assumptions:

(1) $V(x) \rightarrow+\infty$ as $|x| \rightarrow+\infty$;

(2) $1 / V \in L^{1}\left(\mathbb{R}^{n}\right)$;

(3) for every $M>0$ the Lebesgue measure $\mu\left(\left\{x \in \mathbb{R}^{n}: V(x) \leq M\right\}\right)<\infty$.

Each of these conditions guarantee that the space $E:=\left\{u \in W^{1, n}\left(\mathbb{R}^{n}\right)\right.$ : $\left.\int_{\mathbb{R}^{n}} V|u|^{n} d x<\infty\right\}$ is compactly embedded in the Lebesgue space $L^{p}\left(\mathbb{R}^{n}\right)$ for all $p \geq n$.

We point out that just a few results are available when $V(x)$ changes sign, see [11], [26], [31]. However, we stress that in all the above quoted papers, the authors require the potential $V$ to be continuous and bounded below. Here, we concentrate our efforts to treat the case in which $V$ can change sign without requiring any additional condition in order to get compactness. In particular we do not require a uniform bound on the potential which may develop singularities near zero. Physically, this correspond to collision of particle with the center of force, see for instance [22] for more details. Throughout the paper, we assume the following hypotheses on $V$ :

$\left(\mathrm{V}_{1}\right) V: \mathbb{R}^{n} \rightarrow \mathbb{R}$ is measurable and there exist $b_{0}, R_{0}>0$ such that

$$
V(x) \geq b_{0}, \quad \text { for }|x| \geq R_{0} ;
$$

$\left(\mathrm{V}_{2}\right) V^{-} \in L^{p}\left(B_{R_{0}}\right)$ for some $1<p \leq \infty$;

where $V^{ \pm}=\max \{ \pm V, 0\}$ and $B_{R}$ denotes the open ball centered at the origin in $\mathbb{R}^{n}$.

A typical example of function which satisfies the hypotheses $\left(\mathrm{V}_{1}\right)-\left(\mathrm{V}_{2}\right)$ is given by

$$
V_{\varepsilon}(x)= \begin{cases}1+\eta(x) & \text { for }|x| \geq 1, \\ -\frac{\varepsilon}{|x|^{n /(2 p)}} & \text { for }|x|<1,\end{cases}
$$

where $\varepsilon>0, p>1$ and $\eta$ is a nonnegative measurable function for $|x| \geq 1$.

Our second aim in this paper is treat nonlinearities $g(x, s)$ possibly discontinuous and that may exhibit exponential growth. In order to better describe 
the hypotheses on $g(x, s)$ we recall some well known facts about the limiting Sobolev embedding theorem.

Let $\Omega$ be a smooth domain in $\mathbb{R}^{n}$ and $W^{1, p}(\Omega)$ be the Sobolev space endowed with its usual norm

$$
\|u\|_{W^{1, p}(\Omega)}=\left[\int_{\Omega}\left(|\nabla u|^{p}+|u|^{p}\right) d x\right]^{1 / p} .
$$

We define $W_{0}^{1, p}(\Omega)$ as the closure of $C_{0}^{\infty}(\Omega)$ in $W^{1, p}(\Omega)$. After the works [21], [24] and [29], the limiting case $p=n$ of the Sobolev embedding has received considerable attention in recent years. Roughly speaking, their results state that if $\Omega$ is bounded and $\alpha>0$ then there holds the embedding

$$
W_{0}^{1, n}(\Omega) \hookrightarrow L_{A}(\Omega),
$$

where $L_{A}(\Omega)$ is the Orlicz space based on the $N$-function $A(s)=e^{|s|^{n /(n-1)}}-1$. In fact, this embedding is the "best possible" within the Orlicz framework, that is, if there exists any embedding of the form

$$
W_{0}^{1, n}(\Omega) \hookrightarrow L_{B}(\Omega),
$$

then $A$ dominates the $N$-function $B$ near infinity, see [16] (for others improvements, see e.g. [6]). This fact was the main motivation for the notion of exponential critical growth as introduced in the papers [2], [9].

When $\Omega=\mathbb{R}^{n}$, Adams (see [1]) proved that if $n \geq 2, \alpha>0$ and $u \in W^{1, n}\left(\mathbb{R}^{n}\right)$ then

$$
\int_{\mathbb{R}^{n}} \Phi_{\alpha}(u) d x<\infty
$$

where

$$
\Phi_{\alpha}(s):=e^{\alpha|s|^{n /(n-1)}}-\sum_{j=0}^{n-2} \frac{\alpha^{j}|s|^{j n /(n-1)}}{j !} .
$$

This result has motivated the study of semilinear and quasilinear elliptic equations in the whole space $\mathbb{R}^{n}$, when the nonlinearity $g(x, s)$ behaves as $\Phi_{\alpha}(s)$ at infinity, among others see [7], [12], [15], [23] and the references therein.

We emphasize that if $g(x, s)$ is a Carathéodory function, for instance, as in the works [10], [13], [14], [18], [28], [30], one can perform minimization arguments (see for example [20]), based on Ekeland variational principle and Lemma 2.1 of [9] to find a solution for nonhomogeneous problems of the type (1.1).

In this paper, we consider a more general class of nonlinearities $g(x, s)$, namely:

$\left(\mathrm{G}_{1}\right)$ for each $u: \mathbb{R}^{n} \rightarrow \mathbb{R}$ measurable, the Nemytskiu function $N_{g}: \mathbb{R}^{n} \rightarrow \mathbb{R}$, $N_{g}(x)=g(x, u(x))$ is measurable; 
$\left(\mathrm{G}_{2}\right)$ for each $x \in \mathbb{R}^{n}, g(x, s)$ is nondecreasing in $s$ and $|g(x, s)| \leq c_{1} k(x)|s|^{\rho}+c_{2} \Phi_{\alpha_{0}}(s)|s|^{\mu}, \quad$ for all $(x, s) \in \mathbb{R}^{n} \times \mathbb{R}$, where $k \in L^{\sigma}\left(\mathbb{R}^{n}\right)$ for some $1<\sigma \leq \infty, c_{1}, c_{2}>0, \alpha_{0}>0, \rho>n-1$ and $\mu>n-1$.

EXAMPLE 1.1. As an example of a discontinuous function $g(x, s)$ satisfying the conditions above, consider

$$
g(x, s)=\left[k(x)|s|^{\rho-1} s+|s|^{\mu-1} s \Phi_{\alpha_{0}}(s)\right] H(s),
$$

where $k$ is a nonnegative function in $L^{\sigma}\left(\mathbb{R}^{n}\right)$ for some $1<\sigma \leq \infty, \rho>n-1$, $\mu>n-1$ and $H$ is such that $H(s)=1$ if $s<1$ and $H(s)=2$ if $s \geq 1$.

EXAmPle 1.2. Let $g: \mathbb{R}^{n} \times \mathbb{R} \rightarrow \mathbb{R}$ be a locally bounded function, that is, for any bounded interval $I \subset \mathbb{R}$, there exists $C>0$ such that $|g(x, s)| \leq C$ for every $(x, s) \in \mathbb{R}^{n} \times I$. If $g(x, s)$ is nondecreasing in $s$, behaves at infinity as $\Phi_{\alpha}(s)$ and it holds

$$
\lim _{s \rightarrow 0} \frac{g(x, s)}{|s|^{\rho-1} s}=b \in \mathbb{R}, \quad \text { uniformly in } x \in \mathbb{R}^{n},
$$

with $\rho>n-1$, then conditions $\left(\mathrm{G}_{1}\right)-\left(\mathrm{G}_{2}\right)$ are satisfied.

In order to obtain the existence of solutions for (1.1), we work in the subspace of $W^{1, n}\left(\mathbb{R}^{n}\right)$ defined by

$$
X=\left\{u \in W^{1, n}\left(\mathbb{R}^{n}\right): \int_{\mathbb{R}^{n}} V^{+}(x)|u|^{n} d x<\infty\right\}
$$

and we consider in $X$ the norm

$$
\|u\|:=\left[\int_{\mathbb{R}^{n}}\left(|\nabla u|^{n}+V^{+}(x)|u|^{n}\right) d x\right]^{1 / n} .
$$

Using condition $\left(\mathrm{V}_{1}\right)$, we show that the embedding $X \hookrightarrow W^{1, n}\left(\mathbb{R}^{n}\right)$ is continuous (see Lemma 2.1 below). Observing that

$$
\|u\|=\left(\|\nabla u\|_{n}^{n}+\left\|\left(V^{+}(x)\right)^{1 / n} u\right\|_{n}^{n}\right)^{1 / n}
$$

and since $W^{1, n}\left(\mathbb{R}^{n}\right)$ and $L^{n}\left(\mathbb{R}^{n}\right)$ are complete spaces, one can see that $(X,\|\cdot\|)$ is a Banach space. Moreover, using the Clarkson's first inequality (see [5, p. 95]) it follows that $X$ is uniformly convex, and thus reflexive.

Next, for all $n \leq t<\infty$ we set

$$
S_{t}:=\inf _{\substack{u \in X \\ u \neq 0}} \frac{\int_{\mathbb{R}^{n}}\left(|\nabla u|^{n}+V^{+}(x)|u|^{n}\right) d x}{\left(\int_{\mathbb{R}^{n}}|u|^{t} d x\right)^{n / t}} .
$$


It follows from the continuous embedding $W^{1, n}\left(\mathbb{R}^{n}\right) \hookrightarrow L^{t}\left(\mathbb{R}^{n}\right)$ that $S_{t}>0$. In this context, we shall assume that $h \in X^{\prime}$, the dual space of $X$, and we say that $u \in X$ is a weak solution of problem (1.1) if the following holds

$$
\int_{\mathbb{R}^{n}}|\nabla u|^{n-2} \nabla u \nabla v d x+\int_{\mathbb{R}^{n}} V(x)|u|^{n-2} u v d x=\int_{\mathbb{R}^{n}} g(x, u) v d x+\lambda\langle h, v\rangle,
$$

for all $v \in X$, where $\langle\cdot, \cdot\rangle$ denotes the duality pairing between $X$ and its dual $X^{\prime}$.

At this point, in addition to the hypotheses on $V$, we assume the condition

$\left(\mathrm{V}_{3}\right)\left\|V^{-}\right\|_{L^{p}\left(B_{R_{0}}\right)}<S_{t_{0}}$, where $t_{0}:=p n /(p-1)>n$.

Note that the potentials $V_{\varepsilon}$ defined in (1.2) satisfy hypothesis $\left(\mathrm{V}_{3}\right)$ provided $\varepsilon$ is sufficiently small.

The main result in this work can be stated as follows:

TheOREm 1.3. Suppose that $\left(\mathrm{V}_{1}\right)-\left(\mathrm{V}_{3}\right)$ and $\left(\mathrm{G}_{1}\right)-\left(\mathrm{G}_{2}\right)$ hold. Then, there exists $\lambda_{0}>0$ such that for all $0<\lambda \leq \lambda_{0}$, problem (1.1) has a weak solution.

The main features of this class of problems are that they are set in unbounded domains and involve a nonlinear operator (for $n \geq 3$ ). Furthermore, the nonlinearity $g(x, s)$ may develop jump discontinuities in $s$ and behave like $\Phi_{\alpha}(s)$ at infinity. For the authors knowledge the the result is new even for the semilinear case $n=2$.

REMARK 1.4. In certain cases, it is possible to obtain nonexistence results for problem (1.1) when $\lambda$ is large. For example, in addition to the hypothesis $\left(\mathrm{V}_{1}\right)$, if we assume that

$$
\int_{|x| \geq R_{0}} \frac{1}{V(x)^{1 /(n-1)}} d x<\infty
$$

one can show that the embedding $X \hookrightarrow L^{t}\left(\mathbb{R}^{n}\right)$ is compact for each $1 \leq t<\infty$ (see for instance [30, Lemma 2.4]). Consequently, the infimum $S_{t}$ in (1.4) is attained in a nonnegative function $\varphi_{t} \in X$. Hence, in particular for $t=n=2$ we have

$$
\int_{\mathbb{R}^{2}} \nabla \varphi_{2} \nabla v d x+\int_{\mathbb{R}^{2}} V^{+}(x) \varphi_{2} v d x=S_{2} \int_{\mathbb{R}^{2}} \varphi_{2} v d x, \quad \text { for all } v \in X .
$$

By the strong maximum principle, it follows that $\varphi_{2}>0$ in $\mathbb{R}^{2}$. Next, setting

$$
M=\sup \left\{S_{2} r-g(x, r):(x, r) \in \mathbb{R}^{2} \times \mathbb{R}\right\},
$$

if we also assume $0<M<\infty$ and $h \in L^{\nu}\left(\mathbb{R}^{2}\right)$ for some $1<\nu \leq \infty$, with $h \geq 0, h \neq 0$, then there exists $0<\Lambda<\infty$ such that problem (1.1) does not have nonnegative solution for $\lambda>\Lambda$. In fact, if $u$ is a nonnegative solution of (1.1), we have

$\int_{\mathbb{R}^{2}} \nabla u \nabla \varphi_{2} d x+\int_{\mathbb{R}^{2}}\left[V^{+}(x)-V^{-}(x)\right] u \varphi_{2} d x=\int_{\mathbb{R}^{2}} g(x, u) \varphi_{2} d x+\lambda \int_{\mathbb{R}^{2}} h(x) \varphi_{2} d x$. 
Using this, and taking $v=u$ in (1.5) we get

$$
\begin{aligned}
\lambda \int_{\mathbb{R}^{2}} h(x) \varphi_{2} d x=-\int_{\mathbb{R}^{2}} V^{-}(x) u \varphi_{2} d x+\int_{\mathbb{R}^{2}}\left[S_{2} u\right. & -g(x, u)] \varphi_{2} d x \\
& \leq \int_{\mathbb{R}^{2}}\left[S_{2} u-g(x, u)\right] \varphi_{2} d x
\end{aligned}
$$

and therefore we must have

$$
\lambda \leq \Lambda:=\frac{M \int_{\mathbb{R}^{2}} \varphi_{2} d x}{\int_{\mathbb{R}^{2}} h(x) \varphi_{2} d x} .
$$

REmark 1.5. Motivated by the classical Trudinger-Moser inequality, the authors in [2], [12], [9] have introduced the notion of subcriticality and criticality, namely, we say that a function $g(x, s)$ has subcritical growth if for all $\alpha>0$,

$$
\lim _{|s| \rightarrow \infty} g(x, s) e^{-\alpha|s|^{n /(n-1)}}=0 \quad \text { uniformly in } x \in \mathbb{R}^{n},
$$

and has critical growth if there exists $\alpha_{0}>0$ such that

$$
\lim _{|s| \rightarrow \infty} g(x, s) e^{-\alpha|s|^{n /(n-1)}}= \begin{cases}0 & \text { for all } \alpha>\alpha_{0}, \\ +\infty & \text { for all } \alpha<\alpha_{0}\end{cases}
$$

uniformly in $x \in \mathbb{R}^{n}$. We point out that hypothesis $\left(\mathrm{G}_{2}\right)$ includes nonlinearities with this kind of growth.

REMARK 1.6. Using the regularity result due to J. Serrin [25] and (1.3) one can prove that weak solutions to problem (1.1) actually belong to $C_{\text {loc }}^{1, \theta}\left(\mathbb{R}^{n}\right)$.

REMARK 1.7. We mention that recently nonhomogeneous problems involving the critical exponential growth for singular elliptic equations in unbounded domains have been investigated, among others, by J.M. do Ó and M. de Souza [10], N. Lam and G. Lu [18] and Y. Yang [30]. It is important to mention that our results also apply, with slight modification, to the singular case and for a class of more general operators, such as, Leray-Lions operators.

The outline of the paper is as follows: Section 2 contains some preliminary results which are need to the proof of our main result and in Section 3 we prove Theorem 1.3.

Hereafter, $C, C_{0}, C_{1}, C_{2}, \ldots$ will denote positive (possibly different) constants and we shall use the notation $\|\cdot\|_{p}$ for the norm in $L^{p}\left(\mathbb{R}^{n}\right)$.

\section{Preliminary results}

In this section, we obtain some technical results and we establish the appropriate setting to prove Theorem 1.3. 
Lemma 2.1. By condition $\left(\mathrm{V}_{1}\right)$ the space $X$ is continuously embedded in $W^{1, n}\left(\mathbb{R}^{n}\right)$. In particular, the embedding $X \hookrightarrow L^{s}\left(\mathbb{R}^{n}\right)$ is continuous for each $s \in[n, \infty)$.

Proof. It is enough to check that there exists a constant $C>0$ such that

$$
\int_{\mathbb{R}^{n}}\left(|\nabla u|^{n}+V^{+}(x)|u|^{n}\right) d x \geq C \int_{\mathbb{R}^{n}}|u|^{n} d x, \quad \text { for all } u \in X .
$$

Suppose, by contradiction, that (2.1) does not hold. Then for each $k \in \mathbb{N}$ there exists $u_{k} \in X$ such that

$$
\int_{\mathbb{R}^{n}}\left|u_{k}\right|^{n} d x=1 \quad \text { and } \quad \int_{\mathbb{R}^{n}}\left(\left|\nabla u_{k}\right|^{n}+V^{+}(x)\left|u_{k}\right|^{n}\right) d x<1 / k .
$$

So $\left\|\nabla u_{k}\right\|_{n} \rightarrow 0,\left(u_{k}\right)$ is bounded in $W^{1, n}\left(\mathbb{R}^{n}\right)$ and, up to a subsequence,

$$
u_{k} \rightarrow u \quad \text { in } W^{1, n}\left(\mathbb{R}^{n}\right) .
$$

Now, by using the Gagliardo-Nirenberg inequality (see [17, Proposition 8.12]) we reach

$$
\left\|u_{k}\right\|_{2 n} \leq C\left\|\nabla u_{k}\right\|_{n}^{1 / 2}
$$

from which it follows that $\left\|u_{k}\right\|_{2 n} \rightarrow 0$. On the other hand, by (2.2) $u_{k} \rightarrow u$ in $L^{2 n}\left(B_{r}\right)$ for all $r>0$. Thus, $u=0$. By $\left(V_{1}\right)$ we have

$$
\int_{|x| \geq R_{0}}\left|u_{k}\right|^{n} d x \leq \frac{1}{b_{0}} \int_{|x| \geq R_{0}} V^{+}(x)\left|u_{k}\right|^{n} d x<\frac{1}{k b_{0}} \rightarrow 0 .
$$

From this convergence and since $u_{k} \rightarrow 0$ in $L^{n}\left(B_{R_{0}}\right)$, taking the limit as $k \rightarrow \infty$ in the equality

$$
1=\int_{\mathbb{R}^{n}}\left|u_{k}\right|^{n} d x=\int_{B_{R_{0}}}\left|u_{k}\right|^{n} d x+\int_{|x| \geq R_{0}}\left|u_{k}\right|^{n} d x,
$$

we obtain a contradiction. Therefore, (2.1) holds and the lemma is proved.

We next recall a version of the Trudinger-Moser inequality which holds in the whole space for which we refer to [1], [7], [12], [19], [23]. We denote the measure of the $(n-1)$-sphere in $\mathbb{R}^{n}$ by $\omega_{n-1}$ and $\alpha_{n}:=n \omega_{n-1}^{1 /(n-1)}$.

Lemma 2.2. Let $n \geq 2$ and $u \in W^{1, n}\left(\mathbb{R}^{n}\right)$. If $\|\nabla u\|_{n} \leq 1,\|u\|_{n} \leq M$ and $0<\alpha<\alpha_{n}$, then there exists a constant $C=C(n, M, \alpha)$ such that

$$
\int_{\mathbb{R}^{n}} \Phi_{\alpha}(u) d x \leq C(n, M, \alpha) .
$$

As a consequence of (2.3), we establish in our function space framework $X$ an estimate that will be essential in our arguments. 
Lemma 2.3. Suppose that $\left(\mathrm{V}_{1}\right)$ holds. Let $u, v \in X$ and $\alpha, \mu>0$. If $\|u\| \leq$ $M$ and $\alpha M^{n /(n-1)}<\alpha_{n}$, then there exists a constant $C=C(\alpha, M, \mu)>0$ such that

$$
\int_{\mathbb{R}^{n}} \Phi_{\alpha}(u)|u|^{\mu}|v| d x \leq C\|u\|^{\mu}\|v\| .
$$

Proof. First, we choose $q>1$ sufficiently close to 1 satisfying

$$
q \alpha M^{n /(n-1)}<\alpha_{n} \text { and } \sigma:=\frac{2 q}{q-1}>\max \{n, n / \mu\} .
$$

Thus, $1 / q+1 / \sigma+1 / \sigma=1$ and applying the generalized Hölder inequality and Lemma 2.1 one has

$$
\begin{aligned}
\int_{\mathbb{R}^{n}} \Phi_{\alpha}(u)|u|^{\mu}|v| d x & \leq\left(\int_{\mathbb{R}^{n}}\left[\Phi_{\alpha}(u)\right]^{q} d x\right)^{1 / q}\|u\|_{\mu \sigma}^{\mu}\|v\|_{\sigma} \\
& \leq C_{1}\left(\int_{\mathbb{R}^{n}} \Phi_{q \alpha}(u) d x\right)^{1 / q}\|u\|^{\mu}\|v\| \\
& \leq C_{1}\left[\int_{\mathbb{R}^{n}} \Phi_{q \alpha M^{n /(n-1)}}\left(\frac{u}{\|\nabla u\|_{n}}\right) d x\right]^{1 / q}\|u\|^{\mu}\|v\|
\end{aligned}
$$

Since $q \alpha M^{n /(n-1)}<\alpha_{n}$, in view of the Trudinger-Moser inequality (2.3) we conclude that

$$
\int_{\mathbb{R}^{n}} \Phi_{\alpha}(u)|u|^{\mu}|v| d x \leq C\|u\|^{\mu}\|v\|
$$

and the proof is finished.

For the convenience of the reader, in the sequel, we recall some basic concepts and notations. Let $X$ be a real Banach space. A nonempty subset $X_{+} \neq\{0\}$ of $X$ is called an order cone if the following hold:

(i) $X_{+}$is closed and convex;

(ii) if $u \in X_{+}$and $\alpha \geq 0$, then $\alpha u \in X_{+}$;

(iii) if $u \in X_{+}$and $-u \in X_{+}$, then $u=0$.

We observe that an order cone $X_{+}$induces in a natural way a partial order in $X$ as follows: $x \preceq y$ if and only if $y-x \in X_{+}$, and $(X, \preceq)$ is called an ordered Banach space. If in addition, $\inf \{x, y\}$ and $\sup \{x, y\}$ exist for all $x, y \in X$ with respect to $\preceq$ then we say that $(X,\|\cdot\|)$ is a lattice. Furthermore, if $\left\|x^{ \pm}\right\| \leq\|x\|$ for all $x \in X$, with $x^{+}:=\sup \{0, x\}$ and $x^{-}:=-\inf \{0, x\}$ then $(X,\|\cdot\|)$ is called a Banach semilattice.

Special examples of Banach semilattices are the Lebesgue spaces $L^{p}(\Omega)$ and Sobolev spaces $W^{1, p}(\Omega)$ ( $\Omega$ is a domain of $\mathbb{R}^{n}$ ) when we consider the natural partial order ( $u \preceq v$ if and only if $u \leq v$ almost everywhere in $\Omega$ ).

Let $(X, \preceq)$ and $(\widetilde{X}, \triangleleft)$ be ordered Banach spaces. We say that an operator $G: X \rightarrow \widetilde{X}$ is increasing if and only if for all $x, y \in X, x \preceq y$ implies $G x \triangleleft G y$. 
A subset $B$ of $X$ is said to have the fixed point property if every increasing operator $S: B \rightarrow B$ has a fixed point.

Now, we present a version of the fixed point result due to S. Carl and S. Heikkilä (see Corollary 2.2 in [8]) which we use to prove Theorem 1.3.

Lemma 2.4. Let $X$ be a Banach semilattice which is reflexive. Then any closed ball of $X$ has the fixed point property.

For more details with respect to definitions and results about ordered Banach spaces, we refer to $[8]$ and references therein.

\section{Proof of Theorem 1.3}

This section is devoted to the proof of Theorem 1.3. To do this, we need to introduce some appropriate operators in order to apply Lemma 2.4. First, we consider the operator $L: X \rightarrow X^{\prime}$ defined by

$$
\langle L u, v\rangle=\int_{\mathbb{R}^{n}}|\nabla u|^{n-2} \nabla u \nabla v d x+\int_{\mathbb{R}^{n}} V^{+}(x)|u|^{n-2} u v d x, \quad u, v \in X .
$$

Note that for each $u \in X, L u$ is a linear map. Moreover, we deduce from Hölder inequality that

$$
|\langle L u, v\rangle| \leq 2\|u\|^{n-1}\|v\|,
$$

which shows that $L u \in X^{\prime}$ and therefore $L$ is well defined. Furthermore, we have

Lemma 3.1. Under the hypothesis $\left(\mathrm{V}_{1}\right)$, the operator $L: X \rightarrow X^{\prime}$ is continuous and invertible.

Poof. Let $\left(u_{k}\right)$ in $X$ such that $u_{k} \rightarrow u$ in $X$. Using Hölder inequality, for $v \in X$ with $\|v\| \leq 1$ we obtain

$$
\begin{aligned}
\left|\left\langle L u_{k}-L u, v\right\rangle\right| \leq & \left.\int_{\mathbb{R}^{n}}|| \nabla u_{k}\right|^{n-2} \nabla u_{k}-|\nabla u|^{n-2} \nabla u|| \nabla v \mid d x \\
& +\left.\int_{\mathbb{R}^{n}} V^{+}(x)|| u_{k}\right|^{n-2} u_{k}-|u|^{n-2} u|| v \mid d x \\
\leq & \left(\left.\int_{\mathbb{R}^{n}}|| \nabla u_{k}\right|^{n-2} \nabla u_{k}-\left.|\nabla u|^{n-2} \nabla u\right|^{n /(n-1)} d x\right)^{(n-1) / n} \\
& +\left(\left.\int_{\mathbb{R}^{n}} V^{+}(x)|| u_{k}\right|^{n-2} u_{k}-\left.|u|^{n-2} u\right|^{n /(n-1)} d x\right)^{(n-1) / n} .
\end{aligned}
$$

Since $u_{k} \rightarrow u$ in $X$, up to a subsequence, $\nabla u_{k} \rightarrow \nabla u$ and $u_{k} \rightarrow u$ almost everywhere in $\mathbb{R}^{n}$. Moreover, there exist $w_{1}, w_{2} \in L^{1}\left(\mathbb{R}^{n}\right)$ such that $\left|\nabla u_{k}\right|^{n} \leq w_{1} \in$ $L^{1}\left(\mathbb{R}^{n}\right)$ and $V^{+}(x)\left|u_{k}\right|^{n} \leq w_{2} \in L^{1}\left(\mathbb{R}^{n}\right)$ almost everywhere in $\mathbb{R}^{n}$. Hence, in view of the Lebesgue dominated convergence theorem and from the last inequality

$$
\left\|L u_{k}-L u\right\|_{X^{\prime}}=\sup _{v \in X,\|v\| \leq 1}\left|\left\langle L u_{k}-L u, v\right\rangle\right| \rightarrow 0
$$


and the continuity of $L$ is proved. Furthermore, from $\langle L u, u\rangle=\|u\|^{n}$ for all $u \in X$ and $n \geq 2$ we get

$$
\lim _{\|u\| \rightarrow \infty} \frac{\langle L u, u\rangle}{\|u\|}=\infty .
$$

On the other hand, using the well known inequality

$$
\left(|a|^{n-2} a-|b|^{n-2} b\right)(a-b) \geq C_{n}|a-b|^{n-2}, \quad a, b \in \mathbb{R}^{n},
$$

one has

$$
\left\langle L v_{1}-L v_{2}, v_{1}-v_{2}\right\rangle \geq C_{n}\left\|v_{1}-v_{2}\right\|^{n}>0
$$

for all $v_{1}, v_{2} \in X, v_{1} \neq v_{2}$. Thus, by the Minty-Browder theorem (see for instance Theorem 5.16 in [5]), the operador $L$ is invertible and this completes the proof.

At this point, we consider another operator $T: X \rightarrow X^{\prime}$ given by

$$
\langle T u, v\rangle=\int_{\mathbb{R}^{n}}\left[V^{-}(x)|u|^{n-2} u+g(x, u)\right] v d x+\lambda\langle h, v\rangle, \quad u, v \in X .
$$

It is clear that for each fixed $u \in X$ fixed $T u$ is a linear map. As a consequence of Lemma 2.3 we have the following estimate:

Lemma 3.2. Assume $\left(\mathrm{V}_{1}\right)-\left(\mathrm{V}_{2}\right)$ and $\left(\mathrm{G}_{1}\right)-\left(\mathrm{G}_{2}\right)$. Let $M>0$ be such that $\alpha_{0} M^{n /(n-1)}<\alpha_{n}$. There exist positive constants $C_{1}, C_{2}$ such that if $\|u\| \leq M$, then

$$
\left.|\langle T u, v\rangle| \leq\left(S_{t_{0}}^{-1}\left\|V^{-}\right\|_{L^{p}\left(B_{R_{0}}\right)}\right)\|u\|^{n-1}+C_{1}\|u\|^{\rho}+C_{2}\|u\|^{\mu}+\lambda\|h\|_{X^{\prime}}\right)\|v\|,
$$

for all $v \in X$. In particular,

$$
\|T u\|_{X^{\prime}} \leq S_{t_{0}}^{-1}\left\|V^{-}\right\|_{L^{p}\left(B_{R_{0}}\right)}\|u\|^{n-1}+C_{1}\|u\|^{\rho}+C_{2}\|u\|^{\mu}+\lambda\|h\|_{X^{\prime}} .
$$

Proof. Here, let us consider $1<p<\infty$ and $1<\sigma<\infty$. The case $p=\infty$ or $\sigma=\infty$ are more simple and they are treated similarly. Note that

$$
\frac{1}{p}+\frac{n-1}{t_{0}}+\frac{1}{t_{0}}=1 \Leftrightarrow t_{0}=\frac{n p}{p-1} .
$$

Since $V^{-}(x)=0$ for $|x| \geq R_{0}$, using the generalized Hölder inequality together with the inequality $\|u\|_{t_{0}}^{n} \leq S_{t_{0}}^{-1}\|u\|^{n}$ one has

$$
\left.\left|\int_{\mathbb{R}^{n}} V^{-}(x)\right| u\right|^{n-2} u v d x \mid \leq S_{t_{0}}^{-1}\left\|V^{-}\right\|_{L^{p}\left(B_{R_{0}}\right)}\|u\|^{n-1}\|v\| .
$$

Analogously, setting $\beta:=\sigma(\rho+1) /(\sigma-1)>n$ we see that

$$
\frac{1}{\sigma}+\frac{\rho}{\beta}+\frac{1}{\beta}=1,
$$

from which follows that

$$
\left.\left|\int_{\mathbb{R}^{n}} k(x)\right| u\right|^{\rho} v d x \mid \leq\|k\|_{\sigma}\|u\|_{\beta}^{\rho}\|v\|_{\beta} \leq C\|k\|_{\sigma}\|u\|^{\rho}\|v\| .
$$


On the other hand, Lemma 2.3 with $\alpha=\alpha_{0}$ yields

$$
\int_{\mathbb{R}^{n}} \Phi_{\alpha_{0}}(u)|u|^{\mu}|v| d x \leq C\|u\|^{\mu}\|v\|
$$

Since $|\langle h, v\rangle| \leq\|h\|_{X^{\prime}}\|v\|$, using estimates (3.2)-(3.4) and condition $\left(\mathrm{G}_{2}\right)$, we obtain the desired result.

Analyzing the previous proof, we observe that (3.2) and (3.3) holds for each $u \in X$ as well as estimate (2.4). This fact together with hypothesis $\left(\mathrm{G}_{2}\right)$ shows that the operator $T$ is well defined.

Now, we define the operator $S: X \rightarrow X$ by $S=L^{-1} \circ T$.

In order to address the existence of a fixed point for $S$, we need the following lemma:

LEMMA 3.3. Under the hypotheses of Theorem 1.3, there exists $R>0$ such that $S\left(B_{X}[0, R]\right) \subset B_{X}[0, R]$, where $B_{X}[0, R]=\{u \in X:\|u\| \leq R\}$.

Proof. Let $u \in X$ and set $v=\left(L^{-1} \circ T\right) u=S u$. Since $\langle L v, v\rangle=\|v\|^{n}$ we get

$$
\|S u\|^{n}=\langle T u, S u\rangle \leq\|T u\|_{X^{\prime}}\|S u\| .
$$

Choosing $R \leq M$, if $\|u\| \leq R$ then by Lemma 3.2 one has

$$
\begin{aligned}
\|S u\|^{n-1} \leq\|T u\|_{X^{\prime}} & \leq S_{t_{0}}^{-1}\left\|V^{-}\right\|_{L^{p}\left(B_{R_{0}}\right)}\|u\|^{n-1}+C_{1}\|u\|^{\rho}+C_{2}\|u\|^{\mu}+\lambda\|h\|_{X^{\prime}} \\
& \leq S_{t_{0}}^{-1}\left\|V^{-}\right\|_{L^{p}\left(B_{R_{0}}\right)} R^{n-1}+C_{1} R^{\rho}+C_{2} R^{\mu}+\lambda\|h\|_{X^{\prime}},
\end{aligned}
$$

which implies

$$
\frac{\|S u\|^{n-1}}{R^{n-1}} \leq S_{t_{0}}^{-1}\left\|V^{-}\right\|_{L^{p}\left(B_{R_{0}}\right)}+C_{1} R^{\rho-n+1}+C_{2} R^{\mu-n+1}+\lambda \frac{\|h\|_{X^{\prime}}}{R^{n-1}} .
$$

Now, choose $R>0$ sufficiently small so that

$$
C_{1} R^{\rho-n+1}+C_{2} R^{\mu-n+1} \leq \frac{1-S_{t_{0}}^{-1}\left\|V^{-}\right\|_{L^{p}\left(B_{R_{0}}\right)}}{2} .
$$

Setting

$$
\lambda_{0}:=\frac{R^{n-1}\left(1-S_{t_{0}}^{-1}\left\|V^{-}\right\|_{L^{p}\left(B_{R_{0}}\right)}\right)}{2\|h\|_{X^{\prime}}},
$$

for all $0<\lambda \leq \lambda_{0}$, we deduce from (3.5) that $\|S u\|^{n-1} / R^{n-1} \leq 1$. Therefore, $S\left(B_{X}[0, R]\right) \subset B_{X}[0, R]$ and the proof is complete.

At this point, we are ready to prove our main result.

Proof of TheOREM 1.3. In order to apply Lemma 2.4, we consider the following partial order in $X$ :

$$
u_{1}, u_{2} \in X, u_{1} \preceq u_{2} \Leftrightarrow u_{1} \leq u_{2} \text { a.e. in } \mathbb{R}^{n} .
$$

It is clear that $(X, \preceq)$ is an ordered Banach space and for all $u, v \in X$, there exist $\sup \{u, v\}$ and $\inf \{u, v\}$ with respect to the order $\preceq$. Moreover, recalling 
that $u^{+}=\sup \{u, 0\}$ and $u^{-}=-\inf \{u, 0\}$, by the order (3.6), $u^{+}$and $u^{-}$are the positive and negative parts of $u$. Since $\left|\nabla u^{ \pm}\right| \leq|\nabla u|$ and $\left|u^{ \pm}\right| \leq|u|$ almost everywhere in $\mathbb{R}^{n}$ we see that $\left\|u^{ \pm}\right\| \leq\|u\|$. Hence $(X, \preceq)$ is a Banach semilattice which is reflexive. We also observe that the dual space $X^{\prime}$, endowed with the order:

$$
\varphi_{1}, \varphi_{2} \in X^{\prime}, \varphi_{1} \triangleleft \varphi_{2} \Leftrightarrow\left\langle\varphi_{1}, v\right\rangle \leq\left\langle\varphi_{2}, v\right\rangle, \text { for all } v \in X_{+}
$$

where $X_{+}:=\left\{v \in X: v \geq 0\right.$ almost everywhere in $\left.\mathbb{R}^{n}\right\}$, is an ordered Banach space.

We claim that $L^{-1}:\left(X^{\prime}, \triangleleft\right) \rightarrow(X, \preceq)$ is an increasing operator. Indeed, let $\varphi_{1}, \varphi_{2} \in X^{\prime}$ such that $\varphi_{1} \triangleleft \varphi_{2}$. Setting $u_{1}=L^{-1} \varphi_{1}$ and $u_{2}=L^{-1} \varphi_{2}$, for $v \in X_{+}$one has

$$
\begin{aligned}
0 \leq\left\langle\varphi_{2}-\varphi_{1}, v\right\rangle= & \left\langle L u_{2}-L u_{1}, v\right\rangle \\
= & \int_{\mathbb{R}^{n}}\left(\left|\nabla u_{2}\right|^{n-2} \nabla u_{2}-\left|\nabla u_{1}\right|^{n-2} \nabla u_{1}\right) \nabla v d x \\
& +\int_{\mathbb{R}^{n}} V^{+}(x)\left(\left|u_{2}\right|^{n-2} u_{2}-\left|u_{1}\right|^{n-2} u_{1}\right) v d x .
\end{aligned}
$$

From this, taking $v=\left(u_{2}-u_{1}\right)^{-} \in X_{+}$and using (3.1) we reach

$$
0 \leq\left\langle\varphi_{2}-\varphi_{1},\left(u_{2}-u_{1}\right)^{-}\right\rangle \leq-C_{n}\left\|\left(u_{2}-u_{1}\right)^{-}\right\|^{n} \leq 0
$$

whence we conclude that $\left(u_{2}-u_{1}\right)^{-}=0$ and so $u_{1} \leq u_{2}$ almost everywhere in $\mathbb{R}^{n}$, that is, $L^{-1} \varphi_{1} \preceq L^{-1} \varphi_{2}$. Now, we prove that $T:(X, \preceq) \rightarrow\left(X^{\prime}, \triangleleft\right)$ is increasing. To this, let $u_{1}, u_{2} \in X$ such that $u_{1} \leq u_{2}$ almost everywhere in $\mathbb{R}^{n}$. Since the function $f(s):=|s|^{n-2} s$ is increasing it follows from assumption $\left(\mathrm{G}_{2}\right)$ that

$$
\begin{aligned}
\left\langle T u_{1}, v\right\rangle & =\int_{\mathbb{R}^{n}}\left[V^{-}(x)\left|u_{1}\right|^{n-2} u_{1}+g\left(x, u_{1}\right)\right] v d x+\lambda\langle h, v\rangle \\
& \leq \int_{\mathbb{R}^{n}}\left[V^{-}(x)\left|u_{2}\right|^{n-2} u_{2}+g\left(x, u_{2}\right)\right] v d x+\lambda\langle h, v\rangle=\left\langle T u_{2}, v\right\rangle,
\end{aligned}
$$

for all $v \in X_{+}$, that is, $T u_{1} \triangleleft T u_{2}$. Consequently, by definition, the operator $S:(X, \preceq) \rightarrow(X, \preceq)$ is also increasing. By Lemma $2.4, B_{X}[0, R]$ has the fixed point property and in view of Lemma 3.3 there exists $u_{0} \in B_{X}[0, R]$ such that $S u_{0}=u_{0}$. Since $S=L^{-1} \circ T$ we have

$$
\left\langle L u_{0}, v\right\rangle=\left\langle T u_{0}, v\right\rangle, \quad \text { for all } v \in X .
$$

Thus, by the definitions of $L$ and $T, u_{0}$ is a weak solution of (1.1) and the theorem is proved.

Acknowledgements. The authors would like to thank the anonymous referee for his/her thorough review and very useful comments/suggestions that improve the clarity and the relevance of this paper. 


\section{REFERENCES}

[1] R.A. Adams, Sobolev spaces, Pure and Applied Mathematics, vol. 65, Academic Press, New York-London, 1975.

[2] Adimurthi, Existence of positive solutions of the semilinear Dirichlet problem with critical growth for the n-Laplacian, Ann. Scuola norm. Sup. Pisa Cl. Sci. 17 (1990), 393-413.

[3] H. Berestycki and P.-L. Lions, Nonlinear scalar field equations I, Existence of infinitely many solutions, Arch. Rational Mech. Anal. 82 (1983), 313-345.

[4] , Nonlinear scalar field equations II, Existence of a ground state, Arch. Rational Mech. Anal. 82 (1983), 347-375.

[5] H. Brezis, Functional analysis, Sobolev spaces and partial differential equations (2011), Universitext. Springer, New York.

[6] H. Brezis and S. Wainger, A note on limiting cases of Sobolev embeddings and convolution inequalities, Comm. Partial Differential Equations 5 (1980), 773-789.

[7] D.M. CAO, Nontrivial solution of semilinear elliptic equation with critical exponent in $\mathbb{R}^{2}$, Comm. Partial Differential Equations 17 (1992), 407-435.

[8] S. Carl and S. Heikkilë, Elliptic problems with lack of compactness via a new fixed point theorem, J. Differential Equations 186 (2002), 122-140.

[9] D.G. De Figueiredo, O.H. Miyagaki And B. Ruf, Elliptic equations in $\mathbb{R}^{2}$ with nonlinearities in the critical growth range, Calc. Var. Partial Differential Equations 4 (1995), 139-153.

[10] M. DE Souza And J.M. Do Ó, On a class of singular Trudinger-Moser type inequalities and its applications, Math. Nachr. 284 (2011), 1754-1776.

[11] Y.H. Ding And A. Szulkin, Bound states for semilinear Schrödinger equations with sign-changing potential, Calc. Var. Partial Differential Equation 29 (2007), 397-419.

[12] J.M. DO Ó, N-Laplacian equations in $\mathbb{R}^{N}$ with critical growth, Abstr. Appl. Anal. 2 (1997), 301-315.

[13] J.M. do Ó, E. Medeiros And U.B. Severo, A nonhomogeneous elliptic problem involving critical growth in dimension two, J. Math. Anal. Appl. 345 (2008), 286-304.

[14] On a quasilinear nonhomogeneous elliptic equation with critical growth in $\mathbb{R}^{N}$, J. Differential Equations 246 (2009), 1363-1386.

[15] J.M. Do Ó AND M.A.S. Souto, On a class of nonlinear Schrödinger equations in $\mathbb{R}^{2}$ involving critical growth, J. Differential Equations 174 (2001), 289-311.

[16] J.A. Hempel, G.R. Morris And N.S. Trudinger, On the sharpness of a limiting case of the Sobolev imbedding theorem, Bull. Austral. Math. Soc. 3 (1970), 369-373.

[17] O. Kavian, Introduction á la théorie des points critiques et applications aux problèmes elliptiques (1993), Springer-Verlag, Paris.

[18] N. LAm AND G. Lu, Existence and multiplicity of solutions to equations of N-Laplacian type with critical exponential growth in $\mathbb{R}^{N}$, J. Funct. Anal. 262 (2012), 1132-1165.

[19] Y. Li AND B. RuF, A sharp Trudinger-Moser type inequality for unbounded domains in $\mathbb{R}^{n}$, Indiana Univ. Math. J. 57 (2008), 451-480.

[20] J. Mawhin and M. Willem, Critical point theory and Hamiltonian systems, Applied Mathematical Sciences, vol. 74, Springer-Verlag, New York, 1989.

[21] J. Moser, A sharp form of an inequality by N. Trudinger 20 (1971), Indiana Univ. Math. J., 1077-1092.

[22] E. NeLson, Feynman integrals and the Schrödinger equation, J. Mathematical Phys. 5 (1964), 332-343.

[23] R. PANDA, Nontrivial solution of a quasilinear elliptic equation with critical growth in $\mathbb{R}^{n}$, Proc. Indian Acad. Sci. Math. Sci. 105 (1995), 425-444. 
[24] S.I. Pohozaev, The Sobolev embedding in the case $p l=n$, Proceedings of the Technical Scientific Conference on Advances of Scientific Research 1964-1965, Mathematics Section, Moscov. Energet. Inst., 1965, pp. 158-170.

[25] J. SerRin, Local behavior of solutions of quasi-linear equations, Acta Math 111 (1964), $248-302$.

[26] B. SiRakov, Standing wave solutions of the nonlinear Schrödinger equation in $\mathbb{R}^{N}$, Ann. Mat. Pura Appl. (4) 181 (2002), 73-83.

[27] W.A. Strauss, Mathematical aspects of classical nonlinear field equations, (Proc. IX G.I.F.T. Internat. Sem. Theoret. Phys., Univ. Zaragoza, Jaca, 1978), Lecture Notes in Physics, vol. 98, Springer, 1979, pp. 123-149.

[28] E. Tonkes, Solutions to a perturbed critical semilinear equation concerning the $N$ Laplacian in $\mathbb{R}^{N}$, Comment. Math. Univ. Carolin. 40 (1999), 679-699.

[29] N.S. Trudinger, On the imbedding into Orlicz spaces and some applications, J. Math. Mech. 17 (1967), 473-484.

[30] Y. YANG, Existence of positive solutions to quasi-linear elliptic equations with exponential growth in the whole Euclidean space, J. Funct. Anal. 262 (2012), 1679-1704.

[31] Z. WANG AND H.S. ZHou, Ground state for nonlinear Schrödinger equation with signchanging and vanishing potential, J. Math. Phys. 52 (2011), 113-704.

Manassés de Souza, Everaldo de Medeiros and Uberlandio Severo

Departamento de Matemática

Universidade Federal da Paraíba

58051-900 João Pessoa, PB, BRAZIL

TMNA : Volume $44-2014-\mathrm{N}^{\mathrm{O}} 2$ 